\title{
COMUNICACIÓN
}

\section{Distribución vertical de zooplancton en El Embalse Arenal, Costa Rica}

\author{
Gerardo Umaña ${ }^{1}$, Federico Villalobos ${ }^{1}$ y Beatriz Bofill. \\ Escuela de Biología. CIMAR Universidad de Costa Rica, 2050 Costa Rica
}

(Recibido 31-I-1996. Corregido 24-IX-1996. Aceptado4-III-1997)

\begin{abstract}
The vertical distribution of Bomina, Daphnia, Ceriodaphnia, Diaptomus and cyclopoids was studied for a 48 hours period in Arenal Reservoir, Costa Rica. In general the zooplankton decreased during day time across the water column. Daphnia, Diaptomus and the cyclopoids had a constant depth distribution with time, whereas Bosmina, and Ceriodaphnia showed slight evidence of vertical migration. Due to the scarcity of planktivorous fishes in the limnetic zone of the reservoir, the migration of small-sized cladocerans is purpoudtely explained by invertebrate predation pressure (e.g. cyclopoids).
\end{abstract}

Key words: Zooplankton, tropics, reservoirs, vertical migration.

La distribución vertical del zooplancton ha sido ampliamente estudiada y se ha notado que tanto en agua dulce como en el mar muestra un comportamiento migratorio (Murtauhg 1985, Vinogradov et al. 1985). Dentro de las hipótesis propuestas para explicar este comportamiento se encuentran la evasión de dedepredadores (Hans-Bernd \& Lampert 1981, Levy 1990), y su relación con el tamaño corporal (Brooks \& Dodson 1965). También por ventajas metabólicas (Lampert et.al. 1988) y beneficios demográficos (Ohman 1990), al moverse a zonas de diferente temperatura. En este trabajo se intenta determinar por primera vez la distribución del zooplancton en la columna de agua en el Embalse del Arenal, Guanacaste, Costa Rica.

El muestreo fue desarrollado por $48 \mathrm{hr}$, en cuatro turnos de muestreo $(6 \mathrm{hr}, 12 \mathrm{hr}, 18 \mathrm{hr}$, $24 \mathrm{hr}$ ) a profundidades de $2,7,12$, y $22 \mathrm{~m}$; en cada profundidad se obtuvieron dos muestras. Estas fueron recolectadas con una trampa de Schindler-Patalas de 30L de capacidad, dotada con una malla de $63 \mu \mathrm{m}$.

El zooplancton se preservó en alcohol etílico al 70\% (Murtaugh 1985). El volumen de las muestras se estandarizó a $20 \mathrm{ml}$. Las muestras se homogenizaron para extraer $1 \mathrm{ml}$ con una pipeta Pasteur. Los organismos se extrajeron del alcohol por centrifugación y se colocaron en una celda de Sedgewick-Rafter con $1 \mathrm{ml}$ de glicerol. En estas submuestras se contaron los cladóceros de los géneros Bosmina, Daphnia, Ceriodaphnia y los copépodos Diaptomus y Cyclopoides totales. Se contaron también los copépodos juveniles y nauplios (incluyendo ambos ordenes) con el fin de tener un amplio espectro de tamaños.

Se realizaron mediciones de penetración de la luz con el disco de Secchi, del oxígeno disuelto y temperatura con un medidor YSI, en cada turno de muestreo y un perfil de Clorofila a, según Strickland y Parsons (1972).

En el sitio de muestreo la profundidad fue de $25 \mathrm{~m}$. Durante el periodo de estudio se observó una leve estratificación de la columna de agua. La temperatura disminuyó gradualmente con la profundidad, de 24 a $25^{\circ} \mathrm{C}$ en la superficie a 23 ${ }^{\circ} \mathrm{C}$ en el fondo (fig. 1A). El oxígeno disuelto también disminuyó gradualmente, 
especialmente a partir de los $10 \mathrm{~m}$ de profundidad, aunque no se observaron condiciones de anoxia total (Fig. 1B). La profundidad de Secchi osciló entre $1.0 \mathrm{~m}$ y 4.0 $\mathrm{m}$, de acuerdo a la hora del día. La clorofila a mostró una distribución vertical con un pico a 7 m (Fig. 1C).

Las larvas Nauplio, Ceriodaphnia y Bosmina fueron los organismos más abundantes en todas las muestras recolectadas. Cada grupo de zooplancton presento una distribución vertical que varió a lo largo del día.

Ceriodaphnia fue más abundante en las profundidades intermedias (7-12 $\mathrm{m})$, sin embargo, mostró cambios con el tiempo (Fig. 2A). En general la densidad fue menor a las $12: 00 \mathrm{hr}$ y mayor entre las $24: 00 \mathrm{hr}$ y las 06:00 hr. Esta disminución al medio día es más marcada a 2 y $7 \mathrm{~m}$ que a 12 y $22 \mathrm{~m}$ de profundidad. Bosmina también prefiere profundidades intermedias aunque la diferencia no es tan marcada (Fig. 2B) y muestra un cambio de densidad con el tiempo, siendo los valores mínimos entre las 06:00 hr y las 12:00 $\mathrm{hr}$. La disminución más fuerte se nota entre los 2 y $7 \mathrm{~m}$ a las 12:00 hr. Daphnia mostró una distribución bastante uniforme con la profundidad, aunque en general la densidad a 2 m es más baja (Fig. 2C). Uno de los días de muestreo se observó un pico a $7 \mathrm{~m}$ a las 12:00 hr que coincide con el pico de clorofila medido ese día. A pesar de ello, no presentó una variación temporal definida como en los dos casos anteriores.

Diaptomus presentó una distribución vertical bastante uniforme, aunque hubo un cambio con el tiempo en toda la columna de agua, con valores mayores entre las 18: $00 \mathrm{hr}$ y 24:00 hr y mínimos entre las 06:00 hr y 12:00 hr (Fig. 2D). Los cyclopoides fueron en general más abundantes entre los 2 y $7 \mathrm{~m}$ de profundidad (Fig. 2E). Los mayores valores corresponden al periodo entre las 18: $00 \mathrm{hr}$ y 24: $00 \mathrm{hr}$, pero los cambios en el tiempo involucraron a toda la columna de agua.

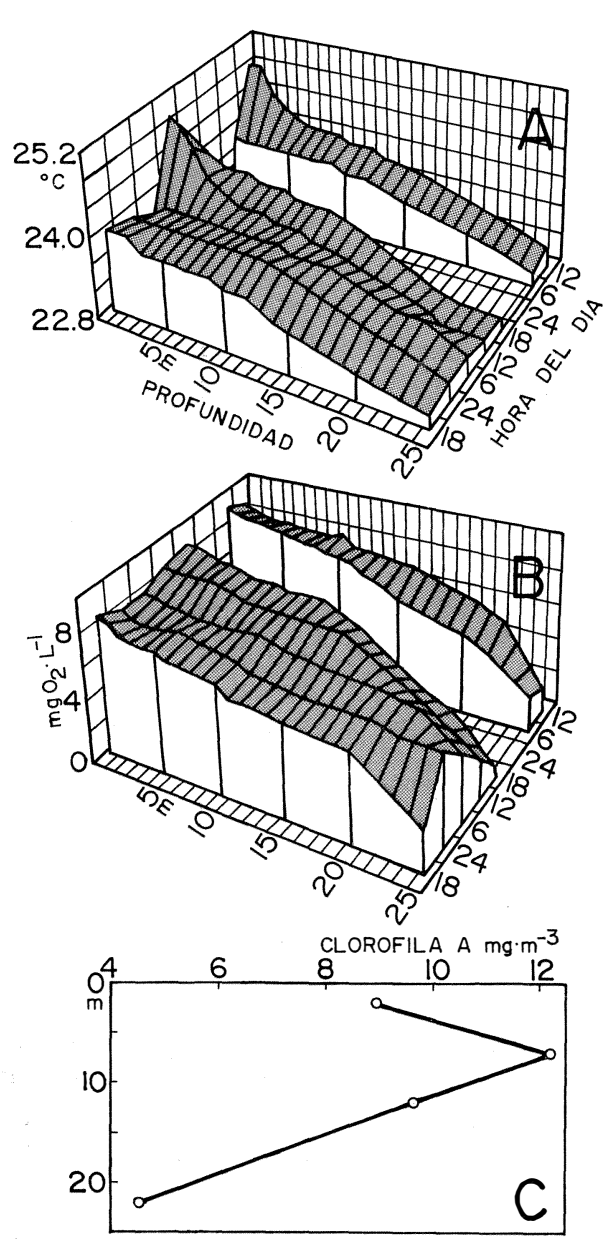

Fig. 1. A) Variación de la temperatura $\left({ }^{\circ} \mathrm{C}\right)$ en el punto de muestreo cercano a la represa, Embalse Arenal, durante el periodo de muestreo. B) Variación del oxígeno disuelto (mg O2 1-1) en el punto de muestreo cercano a la represa, Embalse Arenal, durante el periodo de muestreo. C) Variación vertical de la clorofila a (mg m-3) en el punto de muestreo, Embalse Arenal, el domingo a las 12 medio día.

Los juveniles de copépodos mostraron una distribución bastante uniforme con la profundidad (Fig. 2F). Hubo cambios temporales con máximos a las 18: $00 \mathrm{hr}$ y 
A: Ceriodaphnia
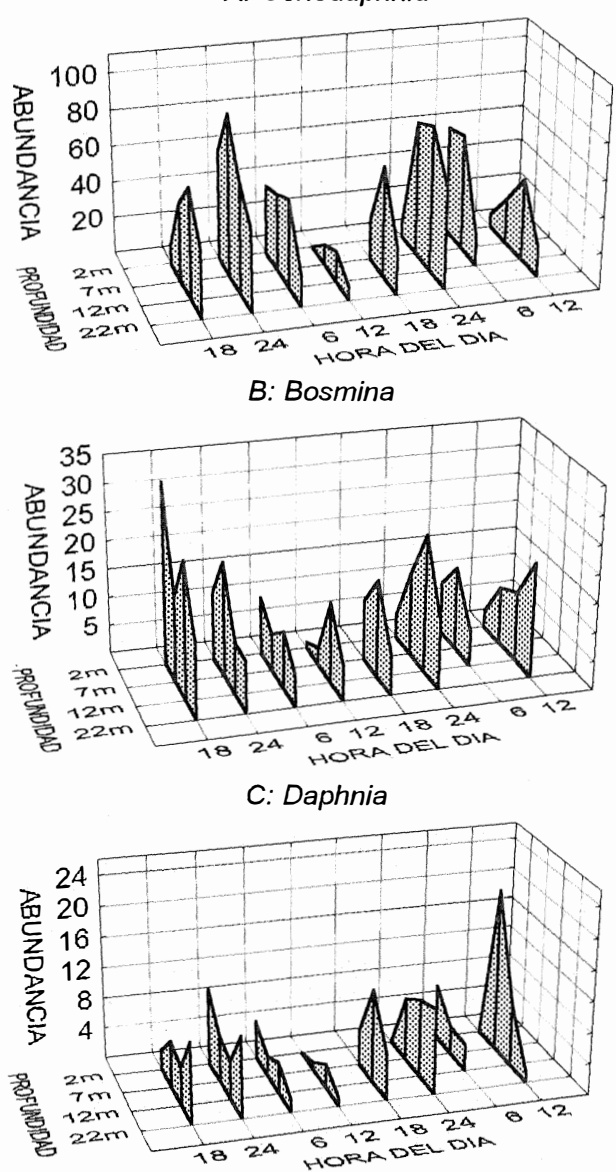

D: Diaptomus

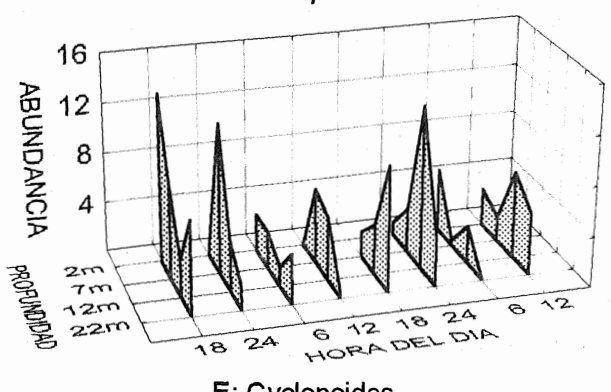

E: Cyclopoides
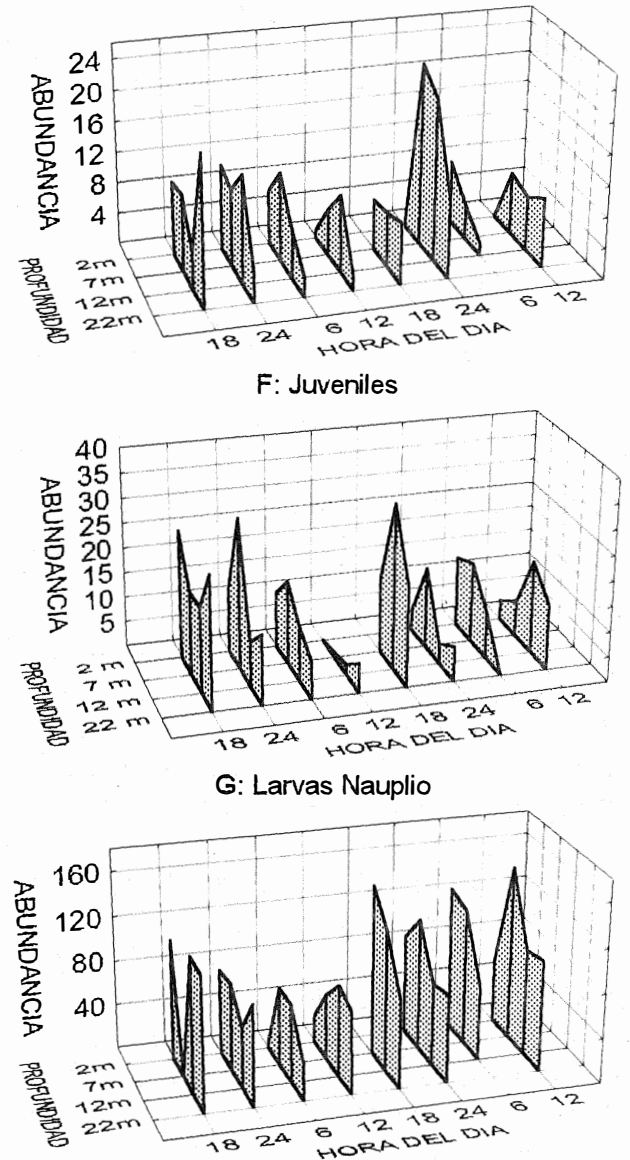

Fig. 2. Variación de la densidad con la profundidad de A) Ceriodaphnia, B) Bosmina C) Daphnia, D) Diaptomus, E) Cyclopoides adultos, F) Copépodos juveniles y G) Larvas nauplio, en el punto de muestreo cercano a la represa, Embalse Arenal, durante el periodo de estudio.

mínimos a las 12: $00 \mathrm{hr}$, en todas las profundidades estudiadas. Las larvas nauplio mostraron una mayor densidad a profundidades intermedias, especialmente a $7 \mathrm{~m}$ (Fig. 2G). Su valor mínimo se observó a $2 \mathrm{~m}$ a las $06: 00 \mathrm{hr}$, pero no hubo un patrón temporal definido.

Los datos sugieren que solamente Bosmina y Ceriodaphnia poseen un patrón de migración 
vertical normal. Los otros grupos no presentaron una distribución vertical que varíe de acuerdo al comportamiento común del zooplancton. En algunos casos la distribución fue casi uniforme.

Los principales factores que pudieron afectar la resolución de la distribución observada fueron: tomar muestras a profundidades muy distantes unas de otras (por ejemplo entre 12 y $22 \mathrm{~m}$ ), los intervalos de $4 \mathrm{hrs}$ entre muestreos, los movimientos de masas de agua y sus cambios en el nivel del oxigeno disuelto (Adeniji 1982) (fig. 1B) como también el efecto de la variación interespecifica en el comportamiento migratorio.

La hipótesis que plantea una mayor eficiencia metabólica y reproductiva por migrar a zonas de mayor temperatura durante la noche no parece aplicarse aquí pues la temperatura mínima no baja de los $23^{\circ} \mathrm{C}$. Mientras que la búsqueda de zonas con más alimento no provocaría necesariamente una migración vertical muy marcada. Pues los organismo se concentrarían donde hubiese mas alimento.

Las especies que migraron fueron los cladóceros de menor tamaño, lo que parece contradecir la hipótesis de Brooks \& Dodson (1965) referente a la selectividad sobre el tamaño de las presas, en donde las especies de mayor tamaño deberían migrar para escapar de los dedepredadores.

Una posible explicación sería que el mayor efecto por depredación se deba a dedepredadores invertebrados, en especial por Cyclopoides, los cuales se sabe que atacan en mayor proporción a cladóceros de tamaño reducido (Gliwicz \& Umaña 1994). Por su origen reciente, el Embalse Arenal no cuenta con una alta densidad de peces en sus aguas abiertas (G.Umaña o.p.). Estos, incluso los planctívoros, se concentran en las orillas. Su ausencia en el área limnética del embalse y la depredación por invertebrados como Cyclopoides puede explicar el comportamiento observado.

\section{AGRADECIMIENTOS}

Este trabajo fue posible gracias al financiamiento de la Vicerrectoría de Investigación de la Universidad de Costa Rica, proyecto no. 808-89-416 y del CONICIT-BID, proyecto no. 89-325-BID. Esta constituye la contribución No. ... del Centro de Investigaciones en Ciencias del Mar y Limnología (CIMAR).

\section{REFERENCIAS}

Adeniji, H.A. 1982. Study of vertical distribution of zooplanton in relation to oxigen and temperature variations in Asa Lake. Annu. Rep. Kainji. Lake Res. Inst. p 25-28. ISSN 0331-9296.

Brooks, J.L. \& S. Dodson. 1965. Predation, body size, and composition of plankton. Science 150: 28-35.

Gliwicz Z.M. \& G. Umaña. 1994. Cladoceran body size and vulnerability to copepod predation. Limnol. Oceanogr. 39: 419-424.

Hans-Bernd, S. 1981. Predator evasion as an explanation of diurnal vertical migration by zooplankton. Nature 293: 396-398.

Lampert, W. R.D. Schmitt \& P. Muck. 1988. Vertical migration of fresh water zooplankton test of some hypotheses predicting a metabolic advantage. Bull. Mar. Sci. 43: 620-640

Levy, D.A. 1990. Reciprocal diel vertical migration behavior in planktivores and zooplankton in British Columbia lakes. Can. J. Fish. Aquat. Sci. 47: 396-398.

Murtavyh, P.A. 1985. Vertical distribution of zooplanton and population dynamics of Daphnia in meromictic lake. Hydrobiologia 123: 47-57.

Ohman, M.D. 1990. The demographics benefits of diel vertical migration by zooplanton. Ecol. Monogr. 60: 257-281.

Vinogradov, M.E. M.V. Flint \& E.A. Shushkina. 1985. Vertical distribution of mesoplankton in the open area of the Black Sea. Mar. Biol. 89: 95-107.

Strickland J.D.H \& T.R. Parsons. 1972. A practical handbook of seawater analysis. Bull. of Fish. Res. Bd. Can. 167. 2ed. p: 1-310.

Umaña, G. \& C. Collado. 1990. Asosiación plantonica en el Enbalce Arenal, Costa Rica. Rev. Biol. Trop. 38 311-321. 